\title{
Delineation of Arboricultural Survelliance with Mobile Media
}

\author{
Syed Umar \\ Professor, Department Of Computer Science Engineering, MLRIT Hyderabad \\ N Priya \\ Assist Professor, Department Of Computer Science Engineering, MLRIT Hyderabad
}

\author{
B. Royyuru Srikanth \\ Assist Professor, Department Of Computer Science Engineering, Institute of Aeronautical Engineering Hyderabad
}

\begin{abstract}
The controlled monitoring of industrial activities that will lead to industry, which will result in damge explode human and industrial property or risk is to overcome it with a new system with lower costeffectiveness of the introduced. This system provides an ideal solution for monitoring critical plant at unmanned sites. Wireless systems are therefore more flexible and cost-effective. Check each site / location for the pre-defined parameters such as temperature, humidity, light, etc. The project will be expanded in the future for additional parameters such as wind speed, etc., and to give warning to agents when a parameter is observed the predetermined value by exceeding the user and give the respective studied. The monitoring kit at each site is designed using the arm, NTC 16x2 dot matrix LCD display, opt coupler and GSM modem, etc., and the information is sent to the central monitoring room or the desired user sent and displayed on an LCD monitor with a buzzer indication. The sensors at different locations are connected to the second channel. When information about the critical condition transmitted by the message, that the area of the situation and the parameters with the cooperation and for solving the critical situation again shows message sent to the user. If the circuit is not successful in all cases, so we have a fail-safe system for regular monitoring circuit.
\end{abstract}

Key Words: Delineation, Arboriculture, Mobile media, Green House

\section{INTRODUCTION}

The industry is the most dynamic in the global greenhouse effect. Environmental separation greenhouse gardening, leading to plants that otherwise would not be able to produce this page. enclosure allows the manipulation of the environment, culture and in the greenhouse. This trumps the farmer in the direction of the desired culture. This higher productivity, longer production times, better quality and less use of protective clothing materials. The effect of the productivity and quality of ingredients portable greenhouse. Make full use of the possibilities raised

Crops and management, it is important to regulate and control the variables in an external system via SMS on GSM - SMS: Dealing With Difficult People almost every day, and the system. Moreover, remote communication system is an important part of the modernization and technology policy, the increasing development of mobile communications. The use of mobile phones, or phones has grown exponentially in recent years. [1], the extension of services to global growth. New wave of growth will come from new technologies (wireless), [2], GSM, it is possible to control and monitor the system length. The main purpose is to measure the low-cost system remote control based on GSM SMS in this article, it is a concept developed by the combination of ARM Greenhouse control [3] and ZigBee module is connected to the serial interface to the GSM network. Various parameter values of effective work remote locations, and each time he crosses the border, the ARM processor to send an SMS to the jurisdiction (s) in your mobile phone. Authority (ies), the system sends a message to the mobile phone system. The advantage of this paper

1. Flexibility / modularity control

2. cheap device that is suitable for different applications. 
3. Adaptable, stable and reliable.

4. An effective and inexpensive way to communicate via SMS.

5. Ideal for monitoring and control of unmanned sites critical of the plant.

\subsection{Manual Set-Up:}

The set-up includes visual inspection of the plant growth, the user water plants, on and off of the temperature control, spraying of fertilizers and pesticides manually. It is time consuming, prone to human error and therefore less accurate and reliable.

\subsection{Semi-Automatic Setup:}

The set-up is a combination of manual control, and respond to partial automation and manual installation in most respects, but it reduces the labor involved in the form of irrigation setup.

\subsection{Completely automated:}

It is an advanced set-up and is well equipped to respond to the most of the climatic changes which take place in the greenhouse. It operates on a feedback system that helps to effectively respond to external stimulus. While this arrangement overcomes the problems that are caused by human error, it is not fully automatic and costly.

\section{LITERATURE SURVEY}

After a series of research projects of greenhouse gas emissions in Israel have found a special company called Top Greenhouses Ltd. is one of the leading offers a wide range of turnkey projects and agriculture solutions for farmers, traders and investors worldwide. The company has an approach to agriculture in the matter: it must be financially, environmentally friendly and efficient, while shortening the distance of the greenhouse to the merchant. As a result, Top Greenhouses to a lot of thinking and invest the design of each project so that it provides the maximum benefit - both in agriculture and economy. Use a staff of experienced professionals in different specialized in the cultivation and agricultural technology, Top Greenhouses works closely with farmers to assess their needs and objectives, conditions and environment for plant growth requirements. Each project is then adjusted to the customer's specifications, budgeted and implemented.

Maintaining the high quality of its projects, the company manufactures its steel products for the construction of greenhouses, with the most advanced technology in the world. The subsidiary Elyahu Zalman \& Sons Ltd. Group, experts in the pipeline design, steel and profiles for more than 50 years, it is useful to Top of vegetable origin with a steady supply of quality raw materials and professional, in-house development of standard and custom assembly that prefabricated and easy to assemble on site. Song Jia [1]. He suggested the supervision and control of the greenhouse system ZigBee wireless sensor network controller 8051. This book is about greenhouse gas monitoring and control system using Zigbee Wireless Network Senor ARM controller and is available to users via the Internet. Ai Wei Chen, CIFA. [2]. They discussed the implementation of environmental monitoring Greenhouse technology is based on the Android platform, the Android platform uses monitoring. In this book, the parameters are checked green home computers, as well as Android mobile phone anywhere in the world when it is connected to the Internet by the team director software. He, Guomi Wang, Xiaochan; Sol Guoxian [3]. They discussed the follow-up of moisture and humidity in greenhouses, where ZigBee. In this article, we have proposed an inexpensive way to use the ARM micro controller and ZigBee technology to accurately track and monitor a number of parameters such as moisture, soil moisture, light intensity and temperature. Aryo H. Primicanta, Nayan Mohd Yunus Awan and Moohammand [4] They proposed method of Zig Bee-based GSM remote reading system. In this article we will use a GSM modem to send error messages to users via ZigBee.

\section{STEPS TO FOLLOW-UP TO CHOOSE THE CONTROL SYSTEM}

In general, three steps can be carried out right Monitoring and control system:

Step \# 1: Define and measure key variables in the industry.

- It is very important to set measurable parameters for the data collection device interface correctly, and how they can be measured.

- A number of variables, which are commonly used for controlling the emission is given below Table 1. Electronic sensor used for measuring the change needs to be available quickly, easily, and cost effectively.

- When the detector is not available, the user in the system, although this is very important.

- In many cases, a change cannot be measured directly continuously or be limited to the system.

- For example, the birth rate in nutrient solutions in greenhouses, it is difficult to measure success. 


\begin{tabular}{|c|c|c|}
\hline Sl. No. & Variable to be monitored & Its Importance \\
\hline 1 & Temperature & Affects all plant metabolic functions. \\
\hline 2 & Humidity & $\begin{array}{r}\text { Affects transpiration rate and the plant's thermal } \\
\text { control mechanisms. }\end{array}$ \\
\hline 3 & Soil moisture & Affects salinity, and pH of irrigation water \\
\hline 4 & Solar Radiation & $\begin{array}{r}\text { Affects photosynthetic rate, responsible for most } \\
\text { thermal load during warm periods }\end{array}$ \\
\hline
\end{tabular}

Step \# 2: Identify leadership skills.

Table:1. Importance of different parameters

- Another important factor in comparison with the control is a strategy that should be followed.

- The easiest way is to use a threshold sensor, which use a direct effect on the actuator.

- For example, exceeds the control temperature in the greenhouse heaters, curtains, and the allowable limits given. Sensor sensing principle is shown in the below figure 1

- The brightness can be set to four positions. As the light intensity decreases the lamp can be activated. And further reduce light output to control the second and so on. This ensures that the plants do not have enough sun in the winter or air.

- More complex control strategies based not only on the current values of variables, but also the history, including the tax system to change settings.

Step \# 3: Determine hardware and software.

It is very important that the operating system functions are set before you decide to purchase the software and hardware. to the model chosen:

- Increase the measured values (data subsystem) and controlled devices (suspension), changing business needs and production growth cannot be met in the future.

- Provide a flexible and easy to use interface.

- In order to ensure the accuracy and durability of the sound. The decor is always a choice of software necessary hardware to support the software selection.

- Moreover, the efficiency, the choice of system should include factors such as reliability, support group experience (positive and negative) and cost

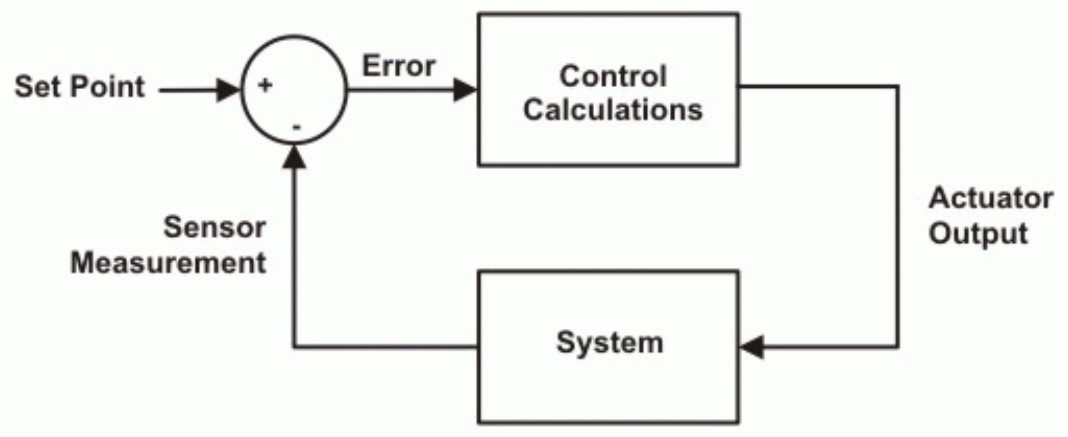

Fig .1: Sensing Principle

\section{SYSTEM PROPOSED FOR CONTROLLED UNIT}

All the major blocks of subsystem included connection for each module consists of a block diagram of temperature sensor, humidity sensor, soil moisture, light sensor, CO2, LPC2148 ARM processor, GSM (SIM300) controls the relay device MAX232 level converter is set to a personal computer. In this application, the system is designed for 
monitoring and regulating the relative unit and ensures a safe operation limit. Detailed Description of the blocks that are set put in the system

\subsection{Advanced RISC Machine V7 LPC2148}

LPC2148 ARM-7 high-performance 32-bit RISC microprocessors expansion Palach 512 kilobytes of programming on-chip Flash ROM System (ISP) and In-Application Programming (IAP), 32 KB of RAM, an interrupt controller, two 10-channel 14 bit ADC, a USB 2.0 full speed device controller, two UART complete modem interface. Two serial interfaces, I2C, SPI, two serial ports, two 32-bit timers, watchdog timer, PWM unit, a real-time clock with an optional battery backup Brown discovered circle Purpose I / O pins CPU clock up to60 MHz crystal oscillator, and PLL on the chip chip. The layout of LPC 2148 is shown in below fig 2. Due to its small size and low power consumption, LPC2148 is ideal for applications where miniaturization is a key requirement, such as access control and point of sale. Serial communication interface of 2.0 full-speed USB device, multiple UART, SPI, SSP I2C bus, and on-chip SRAM $8 \mathrm{~KB}$ to $40 \mathrm{~KB}$ for these devices is very good communication gateways and protocol converters, software modems, voice recognition, and down-market image, which provides a large buffer memory and high performance computing. Several 32-bit timers two 10-bit (s), ADC, 10-bit DAC, PWM-channels, and 45-speed GPIO lines with a maximum of nine external edge or level sensitive interrupt pins of the microcontroller that is capable operating system. [7] [8].

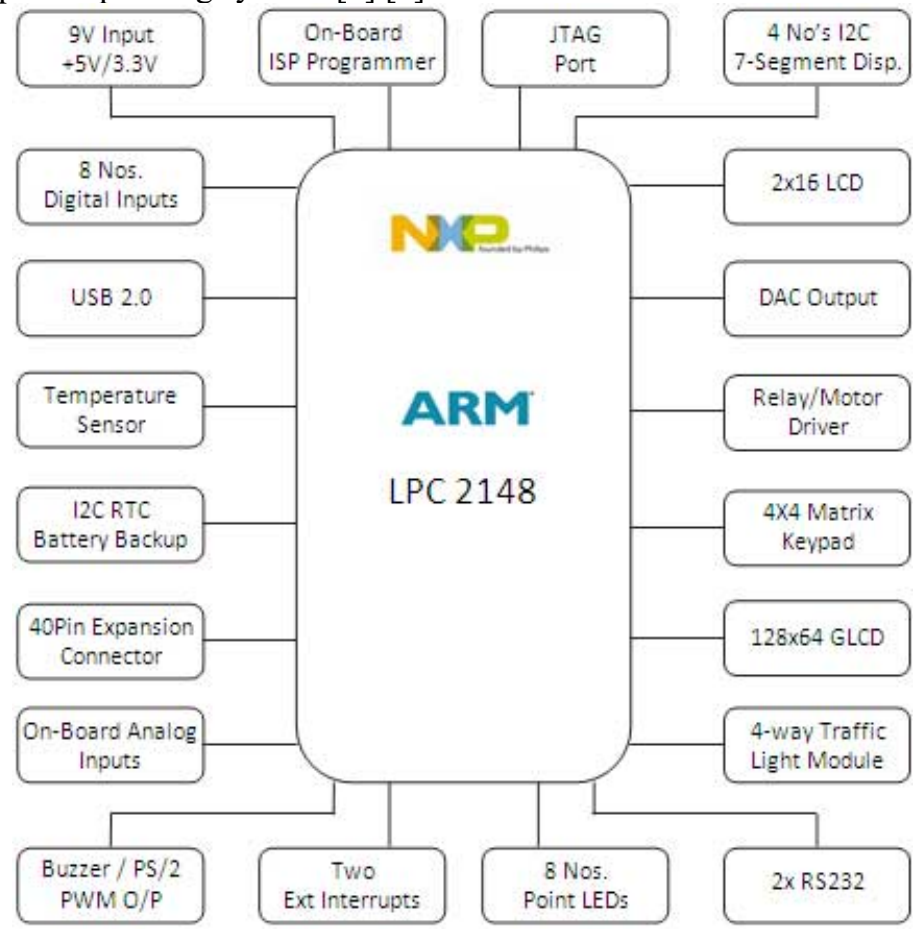

\subsection{Incalescence Sensor.}

Fig 2: Advanced Risc Machine Layout

LM35 series is exactly the Incalescence [temperature] sensors in the integrated circuit, the output voltage is linearly proportional to the temperature in degrees Celsius $\left({ }^{\circ} \mathrm{C}\right)$. LM35 thus has an advantage over linear temperature sensors, because the user is not a large constant output voltage for Celsius scale exercise. Characteristics are as follows. [4]

- The first graduated in degrees Celsius (centigrade)

- Linear scale $+10.0 \mathrm{mV} /{ }^{\circ} \mathrm{C}$

- The accuracy guaranteable $0.5^{\circ} \mathrm{C}\left(+25^{\circ} \mathrm{C}\right)$

- full rated $-55^{\circ} \mathrm{C}$ to $+150^{\circ} \mathrm{C}$ range

- Suitable for outdoor applications

- trim Low-cost wafer level

The seventh task of 4-30 volts humidity sensor (HS-SY-220) The modules converts the relative humidity of the output voltage. The humidity sensor is designed to work with $5 \mathrm{~V} \mathrm{DC}, 0-60^{\circ} \mathrm{c} 30-90^{\circ} \mathrm{C}$ and an output voltage relative humidity of $1980 \mathrm{mV} \pm 25^{\circ} \mathrm{C}$, relative humidity of $60 \%$. [5] 


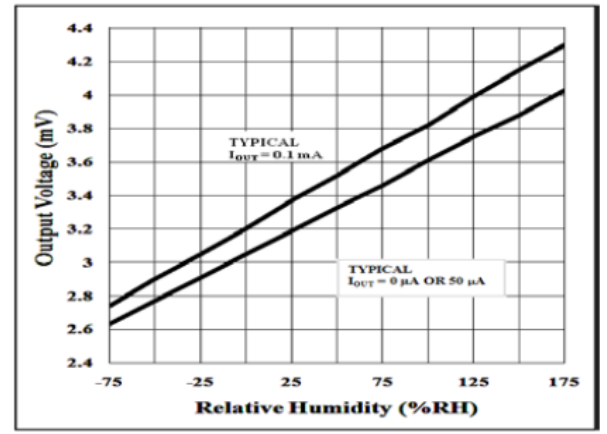

fig .3: Incalescence Calibration

\subsection{VH400 Sensor}

The ground floor consists of two fluid detection sensors cheap (metal rods or just galvanized nails) stood a $\mathrm{t}$ a distance, some insulation. They are used to detect the moist soil. Two pieces of wire, each with 2 "long and $1 / 2 "$ tape end. One end of each wire wound around the head of each nail. Treating a large amount of welding speed wire nails. The resistance of two-finger pinching can be determined in the groundwater in the soil. but the water a higher conductivity .It uses two sensors on the flow by measuring the soil and moisture. But the country will produce conductive (low-resistance), while the conductivity of the dry soil is low (less resistivity)

\subsection{Photo conductivity based LDR [6]}

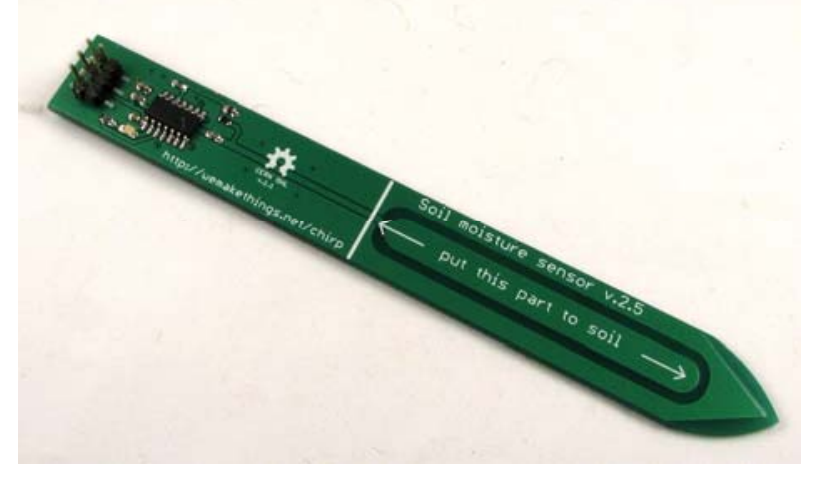

Two cadmium sulfide (CdS), with a spectrum of reactions on the photoconductive human eye. Drug resistance decreases with increasing light intensity. Applications have smoke detectors, automatic lighting control and batch counting and alarm systems.

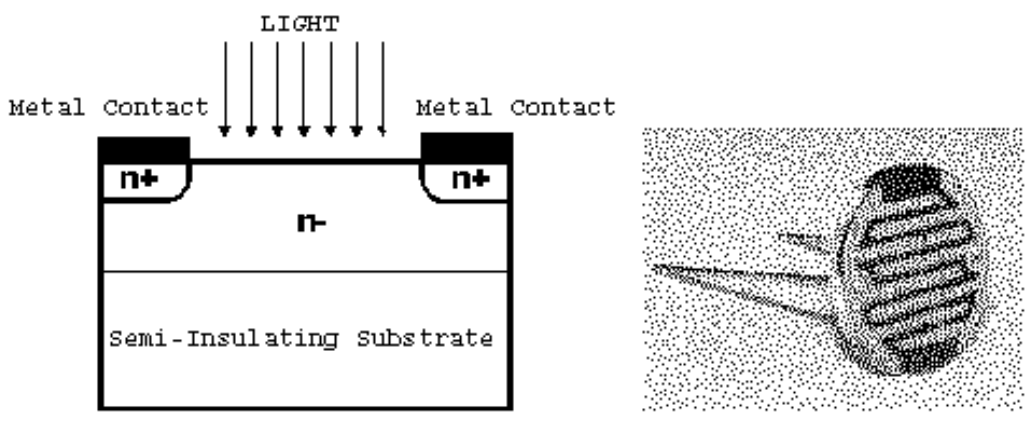

\subsection{GSM modem and PC}

Fig.5 Photo conductive based LDR

GSM modem is a specialized type of modem that accepts a SIM card, and operates over a subscription to a mobile operator, just like a cell phone. From the viewpoint of the mobile operator cellular modem looks like a 
mobile phone. [10] GSM modem can be dedicated to devices with serial, USB or Bluetooth, or perhaps a mobile phone that the functionality of a GSM modem, the modem. Here is the SIM 900A modem. The personal computer is a programmable machine that input, storage receives and manipulates data and produces output in a usable form[11]. Personal computer, a desktop computer, a laptop computer. Capturing data is permanently done LPC2148 ARM7 processor in a personal computer. These data were taken in the software to update the computer database in Visual Basic, and can automatically generate graphs.[12]

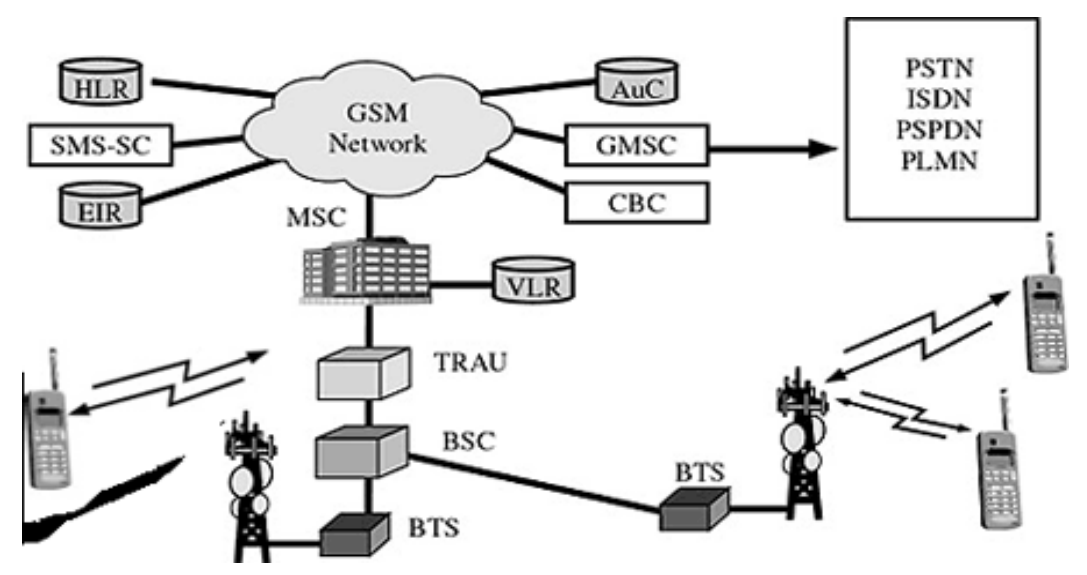

Fig 6. Global System Module Layout

\section{DESIGN OF EQUIPMENT AND SOFTWARE}

The entire system structure shown in Figure . A number of parameters that the temperature, humidity, light, and the sense of providing "the output voltage of the same values.[13] This signal is derived from the reference channels LPC2148 analog data processor. It is digitized with the help of integrated 10-bit ADC data on LPC2148 processor and compared with predetermined data for each change of state or value of those limits. so the system accountable institutions (i) SMS sent via GSM modem / phone consent to a message to send to monitor the microprocessor in / out system. organ (s) ", and you can see the status and needs machine.[14] The measured values are displayed on the PC for further analysis charts. program developed in C integrated Visual Basic. Measurement and control graphs have more variables variable green bone structure: Figure is a part of the control server crisis process. Paper handling two things that need to manage and maintain via the GSM network to the server. To this end, introduced the two checks, after it is of great importance that the message can be found in the GSM system.

This reduces the effective work of a person "spam" junk SMS as a promotional SMS[15] 

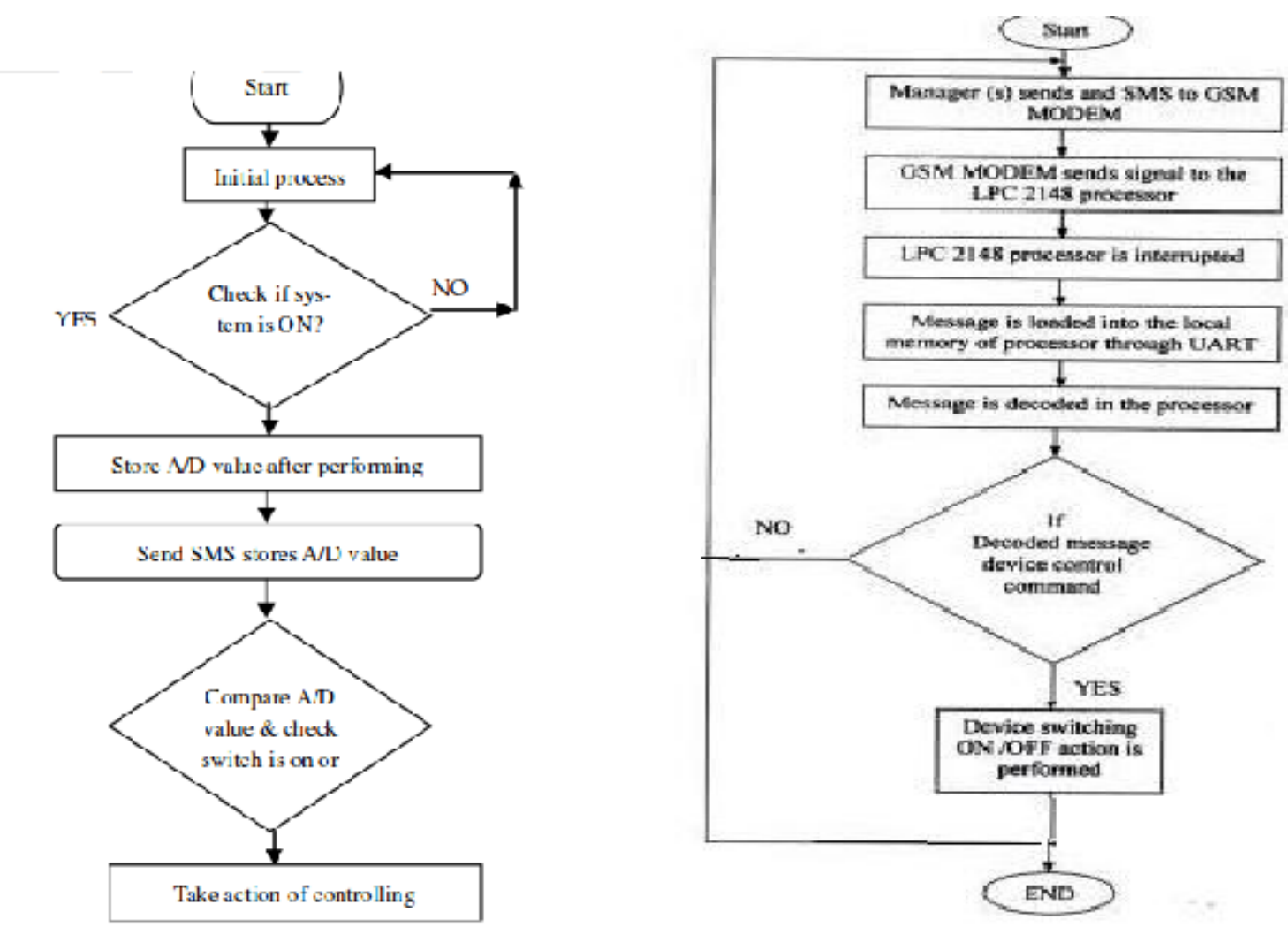

Fig 7. Working flow chart fot the proposed system

\section{CONCLUSION}

The system is designed and run each server using a simple SMS worldwide home via the GSM network for continuous monitoring of Green house. The development process can be a very profitable investment, since they can improve in greenhouses the use of the use of resources. GSM networks operate in the design of this system allows our greenhouse, productive, and to adapt to the current social and economic constraints Morocco is the level of development and economic nations, which correspond consumer society. Our company consists mainly of surgical optimization methods (SMS) to understand the true face climate Morocco parameters and irrigation for agriculture and strengthen the "special breeders, often illiterate. This system, which is a simple SMS, you can start and stop the various actors, as well as knowledge of the greenhouse conditions for GSM networks anywhere.

\section{REFERENCES}

[1] Ken Wieland, "Spreading the World" Telecommunications Magazine International Edition in October 2004. Highlights of numbers.

[2] Ciubotaru Petrescu-B Chiciudean D Cioarga Stănescu R D, "Wireless Telemetry Solutions team monitoring product groups" of three Joint Symposium on Computational Intelligence Applied Romania-Hungary (steal) 25 • May 26, 2006

[3] Trever Martin, stakeholders Guide to Philips ARM7-Based Microcontroller C Eng Miee in February 2005

[4] National Semiconductor Corporation, LM35 specification, actual temperature sensors Celsius, Atmel Databook

[5] SY-HS-220-document [6] http://www.biltek.tubitak.gov.tr/gelisim/elektronik/dosyalar / 40 / LDR_NSL19_M51.pd

[6] Trever Martin, stakeholders Miee Guide Philips ARM7-Based Microcontroller in February 2005

[7] http://www.nxp.com/documentts/data sheet/LPC2148.pdf

[8] The technology Dr.S.S.Riaz Ahamed.Role ZigBee datalink in the future. Journal of Theoretical and Information Technology Application, Vol.5 No. 2, pp 129-135 2005

[9] Puneet Gupta, SMS: How, what and where Developer Network Wireless.

[10] Adedjouma A.Ş., Adjovi G. L. and B. Again Degbo System 2006 Remote control car lock and geographic location, based on GPS and GSM, GSM. African Journal for Research in Computer Science and Applied Mathematics, Vol. 1, p. 1-8. Advantec Co., 1994 PC user manual 812PG LabCard PCL driver software.

[11] Baille A., C. and N. 2001. Impact Kittask Katsoulas greenhouse microclimate laundering and distribution of energy crops. Journal of 
Agricultural and Forest Meteorology, Vol. 107, pp. 293-306.

[12] Benghanem M. 2009. Measurement of meteorological data from the wireless data acquisition system. Journal of Applied Energy, Vol. 86, pp. 2651-2660.

[13] Bouchikhi Eddahhak B. A. Mr. Harzli N. May 2004. The role of sensors and measuring climatic parameters of irrigation water in the greenhouses of the age of science and technology, Cadiz, Tangier

[14] Bouchikhi B. Harzli M. 2005. Design and construction of the acquisition system and climate parameters in the greenhouse. Chema \& Phys. News, Vol. 22, pp. 45-54.

[15] El Fadl of Kherrak A. H., J. and H. Claustriaux Mounhim 1996 Computer Aided greenhouse climate management and the impact on the culture of melons a region of Souss. God Choukr R. (eds.). dog breeding in the Mediterranean region. Mediterranean Notebooks options Vol. 31, pp. 99-108. 